\title{
Les provinces et territoires du Canada devraient publier des données sur le cannabis pour soutenir la recherche
}

\author{
Michael J. Armstrong PhD
}

- Citation : CMAJ 2021 March 8;193:E341-2. doi : 10.1503/cmaj.202041-f

Voir la version anglaise de l'article ici : www.cmaj.ca/lookup/doi/10.1503/cmaj.202041

$\mathbf{L}$

a légalisation du cannabis à usage récréatif au Canada en octobre 2018 a marqué le début d'une expérience politique qui a attiré l'attention du monde entier. Pour appuyer cette expérience, le gouvernement fédéral a financé des recherches médicales, scientifiques et politiques ${ }^{1}$ et s'est engagé à effectuer un examen réglementaire national en octobre $2021^{2}$. Or, la divulgation inadéquate par les gouvernements provinciaux de leurs données sur les ventes de cannabis compromet à la fois les recherches et l'examen. Les provinces et territoires doivent faire preuve de plus de transparence concernant ces données afin de favoriser une compréhension claire des conséquences de la politique canadienne de légalisation du cannabis et des moyens de l'améliorer.

Bien que le gouvernement fédéral réglemente la production de cannabis et les ventes à des fins médicales dans l'ensemble du pays - en exigeant de tous les producteurs titulaires d'une licence qu'ils soumettent des rapports mensuels concernant leurs activités de récolte, de traitement et d'expédition par l'entremise du système de suivi du cannabis et de demande de licences (SSCDL) ${ }^{3}$ de Santé Canada -, les gouvernements provinciaux réglementent la vente de cannabis à usage récréatif sur leur territoire respectif. Chaque province ou territoire dispose d'un organisme désigné, tel qu'Alberta Gaming, Liquor, and Cannabis ou la Société ontarienne du cannabis, qui rend compte à Santé Canada des ventes de cannabis récréatif de la province ou du territoire, tant en termes de dollars que de volume de produits, dans les 15 jours suivant la fin de chaque mois. Cependant, très peu de ces données sont actuellement rendues publiques. Santé Canada publie sur son site Web (https://www.canada.ca/fr/sante-canada/services/drogues -medicaments/cannabis/recherches-donnees/marche.html) le volume total des ventes nationales de produits récréatifs et médicaux, mais seuls les volumes médicaux sont ventilés par province et territoire. Les organismes provinciaux et territoriaux sont moins transparents. Alors que certains publient des rapports de vente trimestriels, plusieurs d'entre eux ne présentent que des rapports annuels, et aucun ne divulgue de données chaque mois.

\section{POINTS CLÉS}

- Malgré les nombreuses conséquences potentielles de la légalisation du cannabis au Canada, les gouvernements provinciaux et territoriaux ne publient pas suffisamment de données sur les ventes de leurs produits à usage récréatif, ce qui entrave les efforts des chercheurs qui souhaitent étudier les effets médicaux et sociaux de la légalisation.

- Aux États-Unis, des États tels que le Colorado et Washington divulguent de manière proactive des données détaillées sur leurs ventes de cannabis, ce qui a permis aux chercheurs de mieux comprendre les conséquences de la légalisation afin d'apporter des améliorations réglementaires.

- Pour que l'examen réglementaire du Canada en 2021 soit équitable et fondé sur des données probantes, les provinces et territoires doivent à tout le moins rendre publiques les données sur les ventes de cannabis récréatif qu'ils recueillent et fournissent déjà à Santé Canada.

Non seulement la publication des données provinciales et territoriales est limitée, mais de nombreux organismes refusent également de répondre aux demandes d'information. D'après un article de recherche de 2020 fondé sur des données gouvernementales pour estimer les ventes mensuelles de cannabis récréatif en dollars par personne, en grammes par utilisateur et en pourcentage de kilogrammes ou de litres consommés ${ }^{4}$, Santé Canada ne pouvait pas publier la ventilation par province sans l'autorisation de cette dernière, et seules 2 provinces ont par la suite communiqué leurs données mensuelles. Les demandes de données des journalistes sont également souvent rejetées. Par exemple, la médecin hygiéniste en chef de l'Alberta a suggéré en juillet 2020 que la consommation de cannabis pourrait avoir augmenté pendant le confinement imposé en 2020 en raison de la pandémie de COVID-19. Alors que les chiffres de vente mensuels de l'Alberta auraient pu corroborer cette hypothèse, Alberta Gaming, Liquor, and Cannabis a refusé de les divulguer (www.cbc.ca/news/canada/calgary/alberta -health-liquor-cannabis-sales-no-actual-data-1.5612664). 
Ce silence de la part des provinces contraste fortement avec les pratiques de divulgation proactives adoptées par plusieurs États américains. Le Colorado publie les ventes mensuelles de cannabis

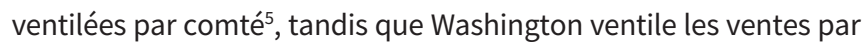
magasin ${ }^{6}$. Ainsi, entre octobre 2018 et mars 2019, le Colorado a mis en ligne 228 points de données sur les ventes au détail et Washington en a fourni 2591, mais l'Ontario n'en a publié qu'un seul. En outre, l'État de Washington permet aux chercheurs d'analyser ses bases de données sur les ventes (anonymisées) qui contiennent des millions de transactions de détail et de gros ${ }^{7}$.

La mise à disposition de données aussi détaillées permet de mieux évaluer les conséquences de la légalisation ${ }^{8}$. Prenons la question réglementaire fondamentale du nombre de magasins autorisés à vendre du cannabis. Un trop petit nombre de magasins pourrait indiquer que les consommateurs continuent à faire affaire avec des revendeurs illégaux, alors qu'un trop grand nombre de magasins pourrait encourager une plus grande consommation. Les rapports détaillés de l'État de Washington ont permis aux chercheurs de mieux comprendre ce problème. Une étude a montré que ce n'est pas la légalisation en soi qui a entraîné une augmentation de la consommation de cannabis, mais plutôt l'ouverture d'un plus grand nombre de magasins ${ }^{9}$. Selon une autre étude, il y aurait trop peu de magasins pour assurer la concurrence, si bien que chaque magasin aurait une influence considérable sur le comportement d'achat de ses clients ${ }^{7}$. À l'heure actuelle, aucune de ces 2 études n'a pu être reproduite dans la plupart des provinces canadiennes.

Par ailleurs, des données détaillées sur les ventes seraient également utiles aux chercheurs en médecine et en sciences sociales. La mesure dans laquelle la légalisation peut réduire les préjudices physiques causés aux consommateurs ou les dommages causés par les forces de l'ordre aux minorités dépend en partie du nombre de consommateurs actuels qui commencent à acheter des produits par l'intermédiaire de canaux réglementés. Les études des effets de la légalisation sur les admissions à l'hôpital et les visites aux services des urgences pourraient notamment bénéficier de données sur les ventes ventilées par mois et par endroit ${ }^{10}$. Mais pour le moment, ces recherches s'appuient plutôt sur des mesures indirectes relatives aux ventes régionales, telles que le dénombrement des magasins autorisés ${ }^{11}$.

Les données détaillées sont également utiles pour la révision des réglementations gouvernementales ${ }^{8}$. La Loi sur le cannabis exige elle-même la tenue d'un examen réglementaire fédéral à compter d'octobre $2021^{2}$. Mais il sera difficile pour les professionnels de la santé, les défenseurs de la justice sociale ou les économistes d'apporter une contribution déterminante si seulement 3 points de données annuels par province sont rendus publics à des fins d'analyse. Les producteurs et les détaillants de cannabis, cependant, seront probablement bien préparés pour l'examen, car ils peuvent s'appuyer sur leurs propres bases de données sur les ventes pour étayer leurs arguments. Un groupe de travail de l'industrie a déjà commencé à préparer une soumission (https://chamber.ca/fr/ committee/groupe-de-travail-national-sur-le-cannabis/?doing_ wp_cron=1614871153.4342620372772216796875) et demandera probablement un assouplissement des règles sur la publicité afin de permettre aux entreprises de " commercialiser leurs produits auprès des personnes qui ne consomment pas de cannabis » (www.thegrowthop.com/cannabis-news/cannabis-beverage -challenge-can-cannabis-beverages-overcome-the-tobaccoization -of-cannabis).

Par conséquent, l'examen réglementaire national risque de compter 3 groupes inégaux : les fonctionnaires fédéraux, qui peuvent accéder aux dossiers détaillés de Santé Canada; les représentants de l'industrie, qui peuvent se fier à leurs propres données; et toutes les autres parties concernées, qui n'ont guère accès à ces données.

Pour que le public comprenne mieux les conséquences de la légalisation du cannabis au Canada et pour éviter un tel déséquilibre, les gouvernements provinciaux et territoriaux doivent faire preuve d'une plus grande transparence concernant leurs données sur le cannabis. À tout le moins, ils devraient commencer à publier en ligne les données mensuelles qu'ils présentent déjà à Santé Canada, dans le même délai de 15 jours. Ces données devraient inclure les ventes totales en dollars et en volume de produits, ventilées par grande catégorie de produits. Idéalement, chaque province devrait aller plus loin en fournissant la ventilation des ventes mensuelles par municipalité ou zone de recensement, comme dans les États du Colorado et de Washington. Les provinces pourraient également inviter les chercheurs à consulter, sous réserve de la protection des renseignements personnels des consommateurs, leurs vastes bases de données sur les ventes.

Une approche gouvernementale ouverte consistant à publier fréquemment des données détaillées sur le cannabis permettrait à un plus grand nombre de parties intéressées de mener leurs propres analyses et de jeter un nouvel éclairage sur cette expérience politique complexe. Les organisations de soins de santé et les autres parties prenantes pourraient ainsi réclamer des améliorations réglementaires fondées sur des données probantes en octobre 2021.

\section{Références}

1. CIHR and health partners invest more than $\$ 21$ million in cannabis research [communiqué de presse]. Ottawa: Canadian Institutes of Health Research; 2020 Nov. 26. Accessible ici : www.canada.ca/en/institutes-health-research/ news/2020/11/cihr-and-health-partners-invest-more-than-21-million-in-cannabis -research.html (consulté le 28 nov. 2020).

2. Cannabis Act, S.C. 2018, c. 16, section 151.1. Accessible ici : https://laws-lois. justice.gc.ca/eng/acts/c-24.5/page-19.html\#h-78291 (consulté le 18 fév. 2021).

3. Cannabis Tracking System: monthly reporting guide. Ottawa: Health Canada; 2020. Accessible ici : www.canada.ca/en/health-canada/services/drugs -medication/cannabis/tracking-system/monthly-reporting-guide.html (consulté le 23 nov. 2020).

4. Armstrong MJ. Legal cannabis market shares during Canada's first year of recreational legalization. Int J Drug Policy 2020;88:103028.

5. Marijuana sales reports: monthly reports. State of Colorado: Colorado Department of Revenue. Accessible ici : www.colorado.gov/pacific/revenue/colorado -marijuana-sales-reports (consulté le 23 nov. 2020).

6. Frequently requested lists. Marijuana sales activity: sales and excise tax by county. Olympia (WA): Washington State Liquor and Cannabis Board. Accessible ici : https://lcb.wa.gov/records/frequently-requested-lists (consulté le 23 nov. 2020). 
7. Hollenbeck B, Uetake K. Taxation and market power in the legal marijuana industry [document de travail]. SSRN 2020 Apr. 25. Accessible ici : https://ssrn. com/abstract=3237729 (consulté le 12 août 2020).

8. Carnevale JT, Kagan R, Murphy PJ, et al. A practical framework for regulating for-profit recreational marijuana in US states: lessons from Colorado and Washington. Int J Drug Policy 2017;42:71-85.

9. Everson EM, Dilley JA, Maher JE, et al. Post-legalization opening of retail cannabis stores and adult cannabis use in Washington State, 2009-2016. Am J Public Health 2019;109:1294-301.

10. Chum A, MacMaster FP. Evaluating the impact of recreational cannabis legalization on acute care outcomes [proposition de subvention de projet]. Ottawa: Canadian Institutes of Health Research; 2020. Accessible ici : https://webapps. cihr-irsc.gc.ca/decisions/p/project_details.html?applld=418978\&lang=en (consulté le 28 nov. 2020).

11. Myran DT, Brown CRL, Tanuseputro P. Access to cannabis retail stores across Canada 6 months following legalization: a descriptive study. CMAJ Open 2019;7:E454-61.

\section{Intérêts concurrents : Aucun déclaré.}

Cet article a été révisé par des pairs.

Affiliation : École de commerce Goodman, Université Brock, St. Catharines, Ont.

Propriété intellectuelle du contenu : Il s'agit d'un article en libre accès distribué conformément aux modalités de la licence Creative Commons Attribution (CC BY-NC-ND 4.0), qui autorise l'utilisation, la diffusion et la reproduction dans tout média à la condition que la publication originale soit adéquatement citée, que l'utilisation se fasse à des fins non commerciales (c.-à-d., recherche ou éducation) et qu'aucune modification ni adaptation n'y soit apportée. Voir : https://creativecommons.org/licenses/by-nc-nd/4.0/deed.fr.

Correspondance : michael.armstrong@brocku.ca 